\title{
Ocorrência de hemoglobinas de efeito Root em peixes amazônicos (*)
}

\author{
Martha Farmer ('); Hans Jorgen Fyhn $\left({ }^{2}\right) ;$ Unni E. H. Fyhn $\left({ }^{2}\right) ;$ Robert W. Noble $\left({ }^{3}\right)$
}

\begin{abstract}
Resumo
O efeito Root foi medido em hemolisados de representantes de 56 gêneros de peixes amazônicos. Hemolisados de váriss espécies de peixes de respiração aérea apresentaram efeito Root, contraria mente às hipóteses publicadas. Hemolisados de Potamotrygon, uma arraia de água doce, exibiram efeito Root sob nossas condiçōes experimentais. O padrão de distribuição do efeito Root correlaciona-se positivamente com a distribuição das retia mirabile da coróide e a distribuição das bexigas natatórias, mas não com a das retia mirabile da bexiga natatória; propõe-se que a primeira é a estrutura mais primitiva que está associada à origem das hemoglobinas com efeito Root. Algumas das hemoglobinas de peixe diferem espectralmente uma das outras. As posiçōes do máximo de absorção das desoxihemoglobinas vão de $553 \mathrm{~nm}$ (Lepidosiren paradoxa e Potamotrygon sp.) a $560 \mathrm{~nm}$ (Plagioscion). Ocorrência de efeitos Root não está corre lacionada com a complexidade do padrão eletroforético da hemoglobina, embora em várias espécies se tenha encontrado sistemas de hemoglobina múltipla, nos quais o efeito Root está restrito a certos componentes.
\end{abstract}

\section{INTRODUÇÃO}

Há, aproximadamente, cinqüenta anos, duas expedições partiram para a América do Sul tropical, a fim de estudar o modo de vida da fauna habitante dos pântanos (Carter \& Beadle 1931; Willmer, 1934). As baixas tensões de oxigênio e condições ácidas freqüentemente encontradas nestas águas paradas resultam em condições respiratórias muito especiais para os habitantes. Carter \& Beadle descobriram que muiots dos peixes haviam adotado hábito de respiração aérea. Eles mostraram que a compo- sição gasosa dos órgãos de respiração aérea destes peixes contém mais que $3 \%$ de díxxido de carbono. Erogh \& Leitch (1919) já haviam demonstrado que $2 \%$ de $\mathrm{CO}_{2}$ eram suficientes não somente para reduzir a afinidade $\operatorname{com~} \mathrm{O}_{2}$ (efeito Bohr), mas também para desoxigenar os sangues de carpa e bacalhau para 52,5 e $32,5 \%$ de saturação de $\mathrm{O}_{2}$ respectivamente. Esta resposta de dessaturação do sangue do peixe ao $\mathrm{CO}_{2}$, ou, mais apropriadamente, ao $\mathrm{pH}$ baixo, veio a chamar-se de efeito Root (Root, 1931), e sua funçảo é aceita como sendo a de facilitar a secreção de $\mathrm{O}_{2}$ dentro da bexiga natatória (Fangë, 1966). Este efeito do $\mathrm{CO}_{2}$ levou Carter $(1931, \mathrm{p} 24)$ a propor mui razoavelmente que o "sangue como é encontrado em peixes típicos de água doce ou marinhos seria então um fluído respiratório impossível para uso em um órgão fechado de respiração aérea. Parece que um passo preliminar para a evolução de respiração aérea nos peixes foi a evolução de um tipo de sangue muito menos sensível à presença de dióxido de carbono... ".

Willmer (1934) investigou a respiraçăo de vários peixes sul-americanos, tanto de respiração aérea como aquática, e seus dados sugeriram que o sangue dos que têm respiração aérea era menos sensível ao $\mathrm{pH}$ baixo, apoiando a hipótese de Carter. Ele mostrou um efeito Bohr muito reduzido para o sangue de Electrophorus electricus e Hoplosternum littorale, e por evidência indireta sugeriu que no sangue de Hoplerythrinus unitaeniatus acontecia o mesmo. Fish (1956) encontrou efeitos Bohr muito pequenos nos sangues de certos peixes africanos de respiração aérea, Clarius mossambicus e Protopterus aethiopicus, também confirmando a hipótese de Carter. Johansen (1966) mostrou que o sangue de Synbranchus

(*) - Versão original inglesa publicada em Comp. Biochem. Physiol. vol. 62A (1). 1979.

(1) - Duke University Marine Laboratory, Beaufort, North Carolina 28516.

(2) - University de Oslo, Institute of Zoophysiology, Blindern-Oslo-3-Norway.

(3) - Department of Medicine and Biochemistry, State University of New York at Buffalo, Veteruns Administration Hospital, Buffalo, New York 14215, Established Investigator of the American Heart Association. 
marmoratus, um teleósteo sul-americano de respiração aérea, tem um pequeno efeito Bohr, mas não tem efeito Root. Ele e colaboradores (Johansen \& Lenfant, 1967a; Lenfant \& Johansen, 1968; e Lenfant, Johansen \& Grigg, 1966) estudaram peixes dipnóicos de três continentes e encontraram um padrão similar: quanto maior a dependência de respiração aérea nas espécies de peixe dipnóico, tanto menor o efeito Bohr. Johansen \& Lenfant (1967b) também estudaram três anfíbios que tendiam a contradizer a relação, uma vez que os efeitos Bohr aumentavam ligeiramente com o aumento de dependência de respiração aérea, mas os efeitos Bohr eram todos bem pequenos neste caso $\left(\Delta \log \mathrm{P}_{50} / \Delta \mathrm{pH}=-0,13 \mathrm{a}-0,29\right)$.

De modo geral, a literatura subseqüente parece validar a hipótese de Carter de que a transição de respiração aquática para respiração aérea correlaciona-se com uma diminuição da influência do $\mathrm{pH}$ na afinidade da hemoglobina pelo oxigênio e sua capacidade de transportar oxigênio. Portanto, foi uma surpresa quando, no decorrer de nossa própria expedição ao Amazonas no R/V "Alpha Helix", notouse que Arapaima gigas, que tem obrigatoriamente respiração aérea, mostrava um pronunciado efeito Root (Galdames-Portus et al., 1978) . A bexiga natatória de Arapaima é muito vascularizada e repartida, e serve como um pulmão, enquanto que no seu parente Osteoglossum, que tem respiração aquática, a bexiga natatória é normal. A tensão de $\mathrm{CO}_{2}$ no sangue de um peixe respirando água é necessariamente menor que quando respirando ar, indo de 4-10 torr no primeiro a mais de 30 torr no último (Rahn \& Garey, 1973). A hipótese de Carter de que uma hemoglobina com efeito Root na presença de tão altas tensões de $\mathrm{CO}_{2}$ resultaria em uma séria redução na capacidade de $\mathrm{O}_{2}$ é baseada em duas suposições: primeira, que $\circ \mathrm{pH}$ e capacidade tampão do sangue são semelhantes em peixes com respiração aérea e aquática, baixos demais para evitar uma acidificação significativa pela alta tensão de $\mathrm{CO}_{2}$, e segunda, que qualquer redução significativa na capacidade de $\mathrm{O}_{2}$ do sangue é prejudicial. É bem possível que os peixes de respiração aérea tenham desenvolvido diversos mecanismos de compensação, e que nenhuma das suposições seja correta.
Vários peixes com respiração aérea, cujos hemolisados não haviam sido examinados previamente quanto à presença de efeito Root estavam disponiveis e então foi possível testar mais além a hipótese de Carter. Além disso, a ausência de um efeito Root foi verificada em vários peixes de respiração aquática e se suspeitou de um padrão de distribuição filogenética ou relacionada com o habitat.

Como havia muito mais espécies disponíveis do que as que poderiam possivelmente ser examinadas com o demorado procedimento tonométrico para construir curvas de equilíbrio de $\mathrm{O}_{2}$, nós examinamos tantos hemolisados quanto possível, com vistas a simples presença ou ausência de efeito Root. Neste artigo, a distribuição observada de efeitos Root em representantes de 56 gêneros de peixes amazônicos é discutida a relação ao hábito respiratório, à presença de bexiga natatória e rete mirabile da coróide e a consideraçöes ecológicas e filogenéticas. Além disso, dados espectrofotométricos, eletroforéticos (Fyhn et al., 1978) e imunológicos (Reichlin \& Davis, 1978) são examinados no que diz respeito a correlações com a ocorrência de efeitos Root.

\section{MATERIAIS E MÉTODOS}

Os espécimes de peixes foram coletados em duas áreas da bacia amazônica durante a expedição de novembro-dezembro com o R/V "Alpha Helix": no rio Solimões, cerca de 50 $\mathrm{km}$ acima de seu encontro com o rio Negro e no rio Negro, cerca de $60 \mathrm{~km}$ acima de Manaus. O período de coleta foi no fim da estação seca, quando o nível da água estava no seu mínimo na área. Os peixes eram em geral obtidos com arrastão de praia e malhadeiras e eram sangrados imediatamente após a coleta no campo ou transportados para o "Alpha Helix" e sangrados dentro de 4-6 horas após a coleta.

As amostras de sangue foram obtidas e preparadas como descrito por Fyhn et al. (1978). Os hemolisados foram armazenados a $5^{\circ} \mathrm{C}$ e testados para um efeito Root no período de 1.7 dias. Os hemolisados eram diluídos com tampão de citrato $0,05 \mathrm{M}, \mathrm{pH} 5,5$, porque citrato é tanto um bom tampão neste $\mathrm{pH}$ como também um forte fator alostérico da hemoglo- 
bina humana (Antonini et al. 1972) e provavelmente de muitas outras hemoglobinas de vertebrados. Efeitos intraeritrocíticos naturais, como fosfatos orgânicos, estavam necessariamente diluídos, e citrato foi empregado intencionalmente para intensificar o efeito a $\mathrm{pH}$ baixo. O método utilizou um espectrofotômetro DB Beckman, com a câmara celular mantida a $20^{\circ} \mathrm{C}$, e uma cubeta de $1 \mathrm{~cm}$. O hemolisado concentrado era pipetado na cubeta e diluído com tampão citrato $0,05 \mathrm{M}$ (mantido a $20^{\circ} \mathrm{C}$ ) até uma densidade ótica correspondente a uma concentração de hemoglobina (tetrâmero) de 10-12 $\mu \mathrm{M}$. O pH final era de aproximadamente 5,5 . Registramos o espectro de $650 \mathrm{~nm}$ a $450 \mathrm{~nm}$. Tampão Tris sólido era adicionado na cubeta para aumentar o $\mathrm{pH}$ para cerca de $8,0-8,5$, permitindo saturação da hemoglobibina a tensões de $\mathrm{O}_{2}$ atmosféricas. Isto resultou em diluição não registrável da amostra de hemoglobina. Novamente, registramos o espectro entre 650 e $450 \mathrm{~nm}$ com o espectro de hehoglobina sobrepondo-se ao espectro registrado em $\mathrm{pH}$ baixo. Uma pequena quantidade de ditionito foi adicionada ao cubeta e o espectro da desoxiemoglobina foi registrado imediatamente sobre os outros dois espectros. $\mathrm{Na}$ maior parte dos casos, os pontos isosbésticos eram excelentes, embora alguns dos hemolisados contivessem metaemoglobina ou precipitassem (reversivelmente) quando submetidas a $\mathrm{pH}$ baixo. Os hemolisados eram examinados tão logo quanto possível, mas amostras mais velhas eram usadas se uma espécie de peixe não era obtida novamente. Amostras com mais de $5 \%$ de metaemoglobina (como determinado espectralmente, Benesch et. al., 1965) foram descartadas. Por causa das variáveis envolvidas, isto é, hemoglobina não fracionada, pequenas quantidades de metaemoglobina, algumas hemoglobinas ácido-lábeis, poderia ser enganoso quantificar a porcentagem de desoxigenação precisamente. Em vez disto, os dados estão divididos em quatro categorias arbitrárias baseadas em porcentagens aproximadas: $\mathrm{O}$, menos que $10 \%$ de desoxigenação; + , 10-20\% de desoxigenação; ++ , $20-40 \%$ de desoxigenação; +++ , mais que $40 \%$ de desoxigenação. Hb A humana fracionada foi examinada como controle, e apresentou menos que $2 \%$ de desoxigenação sob as condições experimentais. Deve-se levar em conta que os espectros, em muitos casos, são na verdade médias de várias hemoglobinas (ver Tabela I) e um pequeno efeito Root no hemolisado pode resultar da presença de uma hemoglobina (ou hemoglobinas) com um grande efeito Root e uma hemoglobina (ou hemoglobinas) mais abundante sem efeito Root.

\section{REsultados E Discussão}

Os resultados são apresentados na Tabela I, com os peixes dispostos de acordo com a posição taxonômica. O fenótipo da hemoglobina, o número de componentes principais da hemoglobina, e o número total de componentes da hemoglobina referem-se aos padrões eletroforéticos encontrados por eletroforese em disco de gel, de acordo com Fyhn et al. (1978). Os efeitos Root sảo classificados de acordo com sua magnitude, como foi descrito na seção de métodos.

Contrariamente à proposição de Carter de que o sangue de peixes de respiraçáo aérea não teria efeito Root, vários peixes de respiração aérea examinados neste estudo apresentaram este efeito. Arapaima gigas, que tem respiração aérea obrigatória, tem um grande efeito Root, como foi mencionado anteriormente. Além disso, Hoplerythrinus unitaeniatus, que tem respiração aérea facultativa, contrariamente à predição de Willmer (1934), tem um grande efeito Root (ver Riggs et al., 1978, esta edição). Hoplosternum littorale (bagre calictiídeo), com respiração aérea obrigatória tem também um pequeno efeito Root (ver Garlick et al., 1978). Entre os bagres loricaríideos, vários parecem ser capazes de respiração aérea acessória, e muitos têm efeitos Root significativos (Tabela I). Hypostomus tem respiração aérea facultativa (Kramer \& Graham, 1976) e um dos seus fenótipos mostrou um efeito Root. Pterygoplichthys e Loricaria mostraram não ser prejudicialmente afetados por períodos prolongados na terra (observaçöes pessoais) e ambos mostraram efeito Root. Exceto os Callichthyidae e Loricariidae, nenhuma das famílias de bagre predominantemente sem respiração aérea mostrou qualquer evidência de hemoglobinas com efeito Root. O gimnotóide com respiração aérea, Gymnotus carapo, tem 


\begin{tabular}{|c|c|c|c|c|c|c|}
\hline Posição taxonômica/espécies & $\begin{array}{l}\text { Fenótipo de } \\
\text { hemoglobina }\end{array}$ & $\begin{array}{l}\text { N.॰ } \\
\text { maior } \\
\text { Hb } \\
\text { comp. }\end{array}$ & $\begin{array}{l}\text { N.o } \\
\text { total } \\
\text { Hb } \\
\text { comp. }\end{array}$ & $\begin{array}{l}\text { Efeito } \\
\text { Root }\end{array}$ & $\begin{array}{c}\text { Bexiga } \\
\text { Natatória }\end{array}$ & \\
\hline \multicolumn{7}{|l|}{ Classe Chondrichthyes } \\
\hline \multicolumn{7}{|l|}{ Subclasse Elasmobranchii } \\
\hline Familia Potamotrygonidae & & & & & não & não \\
\hline \multirow[t]{3}{*}{ Potamotrygon sp. 1} & $\mathbf{I}$ & 2 & 3 & + & & \\
\hline & II & 2 & 5 & + & & \\
\hline & II var. & 2 & 4 & + & & \\
\hline Potamotrygon sp. 2 & I & 3 & 7 & + & & \\
\hline Potamotrygon sp. 4 & $\mathbf{I}$ & 2 & 4 & + & & \\
\hline \multicolumn{7}{|l|}{ Classe Osteichthyes } \\
\hline \multicolumn{7}{|l|}{ Subclasse Sarcopterygii } \\
\hline \multicolumn{7}{|l|}{ Ordem Dipteriformes } \\
\hline Família Lepidosirenidae & & & & & não & năo \\
\hline Lepidosiren paradoxa (a.b.) & I & 1 & 1 & 0 & & \\
\hline \multicolumn{7}{|l|}{ Subclasse Actinopterygii } \\
\hline \multicolumn{7}{|l|}{ Superordem Clupeomorpha } \\
\hline \multicolumn{7}{|l|}{ Ordem Clupeiformes } \\
\hline \multicolumn{7}{|l|}{ Suboräem Clupeoidel } \\
\hline Família Clupeidae & & & & & $\operatorname{sim}$ & $\operatorname{sim}$ \\
\hline Ylisha amazonica & I & 3 & 4 & +++ & & \\
\hline \multicolumn{7}{|l|}{ Superordem Osteoglossomorpha } \\
\hline Familia Osteoglossidae & & & & & $\operatorname{sim}$ & $\operatorname{sim}$ \\
\hline Osteoglossum bicirrhosum & I & 1 & 1 & +++ & & \\
\hline Arapaima gigas (a.b.) & I & 1 & 2 & +++ & & \\
\hline \multicolumn{7}{|l|}{ Superordem Ostariophysi } \\
\hline \multicolumn{7}{|l|}{ Ordem Cypriniformes } \\
\hline \multicolumn{7}{|l|}{ Subordem Characoidei } \\
\hline Familia Characidae & & & & & $\operatorname{sim}+$ & $\operatorname{sim}$ \\
\hline Chalceus sp. & I & 1 & 1 & +++ & & \\
\hline Charax sp. & $\mathbf{I}$ & 2 & 7 & +++ & & \\
\hline \multirow[t]{2}{*}{ Colossoma sp. } & II & 3 & 5 & +++ & & \\
\hline & III & 4 & 6 & +++ & & \\
\hline Mylossoma sp. & I & 2 & 2 & +++ & & \\
\hline Serrasalmus sp. 1 & I & 2 & 4 & +++ & & \\
\hline Serrasalmus sp. & IV & 8 & 3 & $++t$ & & \\
\hline Triportheus sp. & I & 4 & 4 & ++ & & \\
\hline Familia Erythrinidae & & & & & $\operatorname{sim}+$ & $\operatorname{sim}^{\bullet}$ \\
\hline $\begin{array}{l}\text { Hoplias erythrinus } \\
\text { unitaeniatus (a.b.) }\end{array}$ & & & & & & \\
\hline $\begin{array}{r}\text { unitaeniatus (a.b.) } \\
\text { Hoplias malabaricus }\end{array}$ & I & $\begin{array}{l}4 \\
3\end{array}$ & $\begin{array}{l}4 \\
3\end{array}$ & $\begin{array}{l}+++ \\
+++\end{array}$ & & \\
\hline Familia Cynodontidae & & & & & $\operatorname{sim}+$ & $\operatorname{sim}^{\bullet}$ \\
\hline Rhapiodon vulpinas & I & 4 & 4 & +++ & & \\
\hline Família Prochilodontidae & & & & & $\operatorname{sim}+$ & $\operatorname{sim}^{\bullet}$ \\
\hline Prochilodus sp. & I & 1 & 2 & +++ & & \\
\hline Familia Curimatidae & & & & & $\operatorname{sim}+$ & $\operatorname{sim}^{\bullet}$ \\
\hline Curimatus sp. & II & 1 & 2 & ++ & & \\
\hline Leporinus sp. & 1 & 1 & 2 & ++ & & \\
\hline$\rightarrow$ & II & 2 & 2 & +++ & & \\
\hline Rhytiodus sp. & II & 2 & 4 & +++ & & \\
\hline Schizodon sp. & I & 3. & 4 & +++ & & \\
\hline
\end{tabular}


Familia Hemidontidae Hemiodus sp. 1

Subordem Gymnotoidei

Familia Gymnotidae

Gymnotus carapo (a.b.)

Familia Electrophoridae

Electrophorus electricus (a.b.) I

Familia Rhamphichthyidae

Eigenmannia sp. (a.b.?)

Rhamphichthys sp. (3.b.?)

Sternopygus macrurus (a.b.?)

Subordem Siluroidei

Familia Doradidae

Opsodoras sp.

Pseudodoras sp.

Familia Pimelodidae

Brachyplatystoma sp. 1

Brachyplatystoma sp. 2

Pimelodella sp.

Pimelodus sp.

Pinirampus sp.

Pseudoplatystoma sp.

Sorubim lima

Familia Ageneiosidae

Ageneiosus sp.

Familia Hypophthalmidae

Hypophthalmus sp.

Familia Callichthyidae

Hoplosternum littorale (a.b.)

Hoplosternum littorale,

componente II

Família Loricariddae

Hypostomus sp. (a.b.)

Loricaria sp. (a.b.?)

Loricariichthys,

nova espécie (a.b.?)

Parahemiodon sp. (a.b.?)

Pterygoplichthys sp. 1 (a,b.)

Pterygoplichthys sp. 2 (a.b.)

Spatuloricaria sp. (a.b.?)

Sturisoma sp. (a.b.?)

Subfamilia Ancestrinae,

Unidentified espécies

Superordem Atherinomorpha

Ordem Atheriniformes

Familia Belonidae

Potamorrhaphis sp.

Superordem Acanthopterygii

Ordem Synbranchiformes

Familia Synbranchidae

Synbranchus marmoratus (a.b.)

Ordem Peciformos

- Subordem Percoidei

Familia Sciøenidae

Plagioscion sp.

I

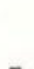

I

I
II

I

I

I

I var.

I var.

III

I

I

I

I

I

I

I

I

V var.

$\mathrm{X}$

I

IV

I

I

I

II

I

I

II

8

naีం

sim

$\operatorname{sim}$

2

3

$+t$

0

$\operatorname{sim}++$

กลัo*

sim ++ năo*

$\operatorname{sim}++\quad$ n.e.

$\operatorname{sim}++$

n.e.

$+$

$+$

0

$+$

$+$

$+$

0

$++$<smiles>CCC</smiles>

$\operatorname{sim}$ sim*

năo*

näo*

nāo*

กลั่

กลีం* 
TABELA 1 (Continuação)

\begin{tabular}{|c|c|c|c|c|c|c|}
\hline Família Cichlidae & & & & & $\operatorname{sim}$ & $\operatorname{sim}$ \\
\hline Acaronia nassa & I & 4 & 4 & +++ & & \\
\hline Aequidens tetramerus & I & 4 & 4 & +++ & & \\
\hline Astronotus ocellatus & II & 5 & 9 & +++ & & \\
\hline Chaetobranchopsis orbicularis & I & 6 & 9 & +++ & & \\
\hline Cinhlasoma severum & I & 5 & 9 & +++ & & \\
\hline Pterophyilum sp. & I & 5 & 8 & +++ & & \\
\hline Ordem Pleuronectiformes & & & & & & \\
\hline $\begin{array}{l}\text { Subordem Soleodei } \\
\text { Familia Soleidae }\end{array}$ & & & & & näo & $\operatorname{sim}^{\bullet}$ \\
\hline $\begin{array}{l}\text { Achirus sp } \\
\text { Ordem Tetraodontiformes }\end{array}$ & I & 1 & 1 & ++ & & \\
\hline Familia Tetraodontidae & & & & & $\operatorname{sim}$ & $\operatorname{sim}$ \\
\hline Colomesus psittacus & I & 2 & 2 & +++ & & \\
\hline
\end{tabular}

TABELA I. Efeitos Root em hemolisados de peixes amazônicos. O esquema filogenético está de acordo com Fink \& Fink (1978). Fenótipos e variantes de hemoglobina e número de componentes de hemoglobina referem-se aos padrões eletroforéticos descritos por Fyhn et al. (1978) A classificação do efeito Root refere-se ao grau em que o hemolisado é desoxigenado a pH 5,5 $20^{\circ} \mathrm{C}$, em tampão de citrato $0,05 \mathrm{M}$ :, menos que $10 \% ;+, 10-20 \% ;++, 20-40 \% ;+++$, mais que $40 \%$. A ocorrência de rete mirabile da bexiga natatória e da coróide é tomada da literatura (Bridge \& Haddon, 1893; Jones \& Marshal, 1953; Alexander, 1965; Greenwood et al., 1966; Nelson, 1976; Wittenberg \& Haedrich, 1974).

um efeito Root, assim como muitos outros gimnotóides cuja capacidade de respiração aérea não foi avaliada. Verificamos no entanto que alguns outros com respiração aérea são desprovidos de efeito Root, por ex., Lepidosiren, Electrophorus e Synbranchus, como já havia sido registrado na literatura (Johansen \& Lenfant, 1967a; Willmer, 1934; Johansen, 1966).

Os diversos peixes com respiração aérea diferem no método de obter oxigênio do ar. Enquanto que os bagres usam porções do intestino intensamente vascularizados para respirar ar, Hoplerythrinus e Arapaima usam ambos bexigas natatórias modificadas como o órgão de respiração aérea (Carter \& Beadle, 1931; Johansen, 1970). Synbranchus respira ar com suas guelras, Electrophorus com o epitélio da boca, altamente vascularizado, e Lepidosiren com um pulmão verdadeiro (Johansen, 1968) . Nenhuma correlação entre órgão de respiração aérea e efeito Root é aparente. Claramente estes órgãos internos de respiração aérea funcionam adequadamente, ainda que $\mathrm{p} \mathrm{CO}_{2}$ seja alta e hemoglobinas com efeito Root estejam presentes. Talvez a reduzida capacidade de oxigênio do sangue não seja uma desvantagem séria, ou seja parcialmente compensada por um aumento na concentração de hemoglobina. Possivelmente também uma alcalose metabólica seja utilizada por estes peixes para compensar a acidose respiratória.

Um segundo achado inesperado foi a descoberta de que as arraias de água doce que nós examinamos, Potamotrygon spp. tinham um efeito Root, embora pequeno, o que é incomum entre os elasmobrânquios. Maxwell $(1958,1963)$ registrou um grau de dessaturação semelhante para soluçöes de hemoglobinas de Squalus suckleyi e Raja binoculatu em tampões de fosfato, mas não considerou isto como sendo um efeito Root, embora isto possa ser uma questão de definiçăo. $O$ sangue de muitos elasmobrânquios carece até de um efeito Bohr (Riggs, 1970). Quatro espécies de Potamotrygon foram apanhadas durante a expedição, e as três examinadas neste estudo tinham todas efeitos Root. Martin et al. (1978a), no entanto, não encontraram efeito Root no espécime de Potamotrygon (espécie 4 , fenótipo II) que eles examinaram por equilibrios tonométricos de oxigênio. Existem três diferenças técnicas que poderiam ter gerado esta discrepância. Primeiro, a pesquisa presente foi efetuada a um $\mathrm{pH}$ mais baixo, 5,5 , ao invés de 6,0 , e segundo, nós usamos tampão de citrato $0,05 \mathrm{M}$ no lugar do bis-tris $/ \mathrm{HCl}$, pre- 
sumivelmente mais inerte (Antonini et. al. 1972). Ambos os fatores poderiam contribuir para decrescer mais a afinidade com o oxigênio da maior parte das hemoglobinas. Além disso, a espécie 4 , fenótipo II, tem um padrão eletroforético surpreendentemente diferente do da espécie 4, fenótipo I, que foi examinada neste estudo (Fyhn et al., 1978).

Classicamente o efeito Root, relatado originalmente por R. W. Root (1931) tem significado simplesmente uma redução na capacidade de oxigênio do sangue quando exposto a condiç̧ões ácidas na presença de tensões de oxigênio atmosféricas. Nenhum padrão foi estabelecido na literatura subseqüente para limites inferiores de desoxigenação, efeitos alostéricos, temperatura ou $\mathrm{pH}$. Mesmo a $\mathrm{Hb}$ A humana pode ser induzida a se comportar um pouco como uma hemoglobina com efeito Root (na presença de IHP 100 vezes em exces. so $\mathrm{pH} 5^{\circ}, 30^{\circ} \mathrm{C}$ ), como nós observamos. Já que a ligação do oxigênio com a hemơglobina é um processo assintótico, saturação de $100 \%$ teoricamente nunca é alcançada, somente aproximada. Existe na realidade uma constante entre o efeito Bohr e o efeito Root que está evidente em nossos dados, e qualquer ponto de ruptura é necessariamente arbitrário. Nossa distinção entre hemoglobinas com e sem efeito Root é baseada nos espectros de $\mathrm{Hb}$ A registrados como controle. Hb A não é desoxigenada mais que $2 \%$ sob as condições experimentais, como pode ser visto na Fig. 1, A. Como foi mencionado na seção de metodologia, menos que $10 \%$ de desoxigenaçăo sob nossas condições experimentais foram consideradas um resultado negativo.

Espectros para Lepidosiren paradoxa, Potamotrygon (sp. 4, fenótipo I), Sternopygus macrurus, Hoplosternum littorale (hemolisado e componente II isolado), Achirus sp. e Plagioscion sp. são mostrados na Fig. 1, B-H, ilustrando a continuidade entre efeitos Bohr e Root.

Uma variável não controlada nestes experimentos é a efetividade dos íons de citrato em reduzir a afinidade, ou capacidade, de oxigên'o, que pode variar entre as hemoglobinas. Em concentração experimental, citrato pode ter sido mais efetivo em algumas soluções diluídas de hemoglobina do que o é o fator intraeritrocítico natural no sangue integral. Uma vez que não se sabe a que $\mathrm{pH}$ o sangue é submetido na rete mirabile da coróide de nenhum dos peixes examinados, é impossivel dizer se um pequeno efeito Root ( + na nossa classificação) sempre afeta a capacidade de $\mathrm{O}_{2}$ ou é funcionalmente apenas um efeita Bohr forte. Além da rete mirabile, no entanto, existe um possível papel para o efeito Root/efeito Bohr em aumentar a liberação de $\mathrm{O}_{2}$ para tecidos com taxa metabólica especialmente alta ou concentrações de matabólitos ácidos. No caso de Potamotrygon, onde nenhuma das retia está presente, este é um papel potencial para o efeito Root.

O padrão de ocorrência do efeito Root (Tabela I) segue grosseiramente o esquema filogenético (ver Fink \& Fink, 1978). Espécimes das ordens primitivas dos Clupeitormes e Osteoglossiformes exibem grandes efeitos Root. Hemolisados tanto de caracóides como de percóides também mostram grandes efeitos Root, enquanto hemolisados da maioria das famílias de Siluróides são desprovidos deste efeito. O efeito Root nos hemolisados de Gimnotóides varia, indo de ausente até um efeito pronunciado. Como foi visto na Tabela 1, peixes amazônicos com uma bexiga natatória bem desenvolvida geralmente têm um efeito Root pronunciado. A mesma correlação geral existe entre o efeito Root e a presença de uma rete mirabile da coróide, nos olhos, embora não com a presença de uma rete mirabile na bexiga natatória. A ocorrência da bexiga natatória nos diversos peixes foi tomada da literatura (Bridge \& Haddon, 1894; Jones \& Marshall, 1953; Alexander, 1967; Greenwood et al., 1966; Nelson, 1976). Esta informação se aplica às diversas familias ou em alguns casos a subordens, uma vez que informação específica sobre os gêneros e espécies examinados só raramente é disponível. A ocorrência de uma rete na coróide em algumas famílias (marcadas com um asterisco na Tabela 1) é deduzida de informação sobre outras famílias da mesma subordem ou ordem como foi dado por Wittenberg \& Haedrich (1974), uma vez que informação específica novamente não é disponível. A suposição de que gêneros estreitamente relacionados mos- 
trarão um padrão semelhante de ocorrência de rete na coróide é fortemente apoiada por seus dados.

Altas tensões de oxigênio são geralmente encontradas em bexigas natatórias (ver revisão por Fanguë, 1966) e no humor vítreo do olho de teleósteos (Wittenberg \& Wittenberg, (1974). Através das semelhanças estruturais das duas retia, Wittenberg \& Wittenberg (1974) propuseram que a rete mirabile da coróide funciona do mesmo modo que a rete $\mathrm{ml}$ rablle da bexiga natatória. Um mecanismo multiplicador de contra-corrente dá como resultado a concentração do oxigênio que é liberado da hemoglobina por acidificação do sangue (Steen, 1963; Kuhn et al., 1963). Hemoglobinas com efeito Root poderiam estar intimamente envolvidas neste processo, por intensificar a transferência do oxigênio do sangue para o lumem da bexiga natatória ou do olho. No caso de caracóides por exemplo, que têm uma bexiga natatória bem desenvolvida, mas como outros ostariofísios, não possuem a rete mirabile da bexiga natatória (Bridge \& Haddon, 1893 : 293), a secreção de oxigênio precisa ser completada sem o efeito concentrador da rete. Um forte efeito Root em peixes que têm uma rete na coróide, mas carecem da rete da bexiga natatória, como é visto entre os caracóides, clupeídeos (Fahlén, 1967a) e a maior parte dos salmonídeos (Fangë, 1958; Fahlén, 1967b), sugere que a rete mirabile da coróide pode ser a característica mais primitiva associada à origem das hemoglobinas com efeito Root. Isso poderia funcionar comc uma pré-adaptação para a evolução de um eficiente mecanismo de secreção de gás na bexiga natatória. Os peixes antes mencionados são mais primitivos que os acontopterígios, onde ambas as retia são geralmente encontradas. Lepidosiren paradoxa, o mais primitivo peixe ósseo encontrado, năo tem nenhum tipo de rete, nenhuma bexiga natatória, e nenhum efeito Root. Por outro lado, exclusivo de água doce, Achirus, que perdeu secundariamente a bexiga natatória, mas tem uma rete na coróide, um efeito Root é encontrado. Não foi determinado se os bagres com efeitos Root têm uma rete na coróide, mas não é improvável que uma característica possa ser perdida sem perda da outra. Eigenmannia por exemplo, não tem qualquer tipo de rete mlrabile, mas tem um apreciável efeito Root.

Entre os ostariofísios (Tabela 1), o efeito Root é forte nos caracóides que têm uma bexiga natatória bem desenvolvida e uma rete na coróide, é variável nos gimnotóides que têm uma bexiga natatória mas presumivelmente não têm rete na coróide, e é pequeno ou ausente nos silurỏides que têm bexigas natatórias reduzidas e geralmente encapsuladas (Bridge \& Haddon, 1893) sendo que os examinados também carecem de rete na coróide. $O$ desenvolvimento do altamente especializado aparelho weberiano em peixes ostariofísios tornou a bexiga natatória importante para audlção. (Poggendorf, 1952), bem como para flutuabilidade. Foi provavelmente a importância do aparelho weberiano que impediu a perda da bexiga natatória em siluróides que vivem no fundo, já que ela comumente foi perdida em outros peixes habitantes do fundo (Alexander, 1967). Nas famílias de bagres encouraçados, Callichthyidae e Loricarlidae, às quais as espécies de respiração aérea pertencem, a bexiga natatória é minúscula, e encapsulada em uma bainha óssea (Alexander, 1966). Pode-se deduzir que a capacidade para secreção e absorção de gás na bexiga natatória, especialmente na ausência da rete mirabile, deve ser muito limitada e de pouca importância nos ajustamentos para flutuação, já que hemoglobinas com efeito Root são encontradas em ambas as famílias. Possivelmente a rete da corb́ide não foi perdida nestas duas famílias, mas os dados não são ainda disponíveis.

Em conjunto, o padrão de ocorrência de efeito Root nos bagres é o contrário do que seria de esperar-se de acordo com a hipótese de Carter de que a respiração aérea é incompativel com a presença de efeito Root.

Talvez o efeito Root e a rete mirabile da coróide tenham sido secundariamente perdidas por estes peixes, geralmente habitantes de pântanos, que não desenvolveram a opção da respiração aérea porque eles precisam ainda estar aptos a extrair $\mathrm{O}_{2}$ de águas que são freqüentemente pouco oxigenadas e acidificadas. Hemolisados de Arapaima gigas e Hoplerythrinus unitaeniatus mostram também grandes efeitos Root; as espécies próximas, Osteoglossum bicirrhosum e Hoplias malabaricus, res- 
pectivamente, que não têm respiração aérea, mas têm efeitos Root semelhante, morrem, se impedidas de alcançar a camada superficial mais oxigenada de tal água (Lowe-Mc Connell, 1975) .

O estudo imunológico de Reichlin \& Davis (1978) mostra uma correlação entre a ocorrência de efeitos Root nos bagres siluróides e a reação imunológica de seus hemolisados. Os hemolisados de siluróides com efeito Root, todos de peixes com respiração aérea, foram os únicos hemolisados de siluróide que precipitaram com anti-soro para componente I da hemoglobina da truta; uma hemoglobina sem efeito Root. Hemolisados de caracóides e gimnotóides, também Ostariophysii, precipitaram do mesmo modo com este anti-soro. Isto sugere que a superfície das moléculas de hemoglobina com efeito Root dos siluróides assemeIha-se tanto à superfície do componente I do sistema de hemoglobina da truta, como à superfície de componentes da hemoglobina de outros ostariofísios. Aparentemente, reatividade cruzada entre duas hemoglobinas pode indicar parentesco evolucionário refletido em certas propriedades da superfície, mas se a base estrutural para o efeito Root altera as propriedades de superfície, esta análise não o detecta.

Os espectros dos hemolisados de diferentes peixes divergem um pouco dos espectros para $\mathrm{Hb} \mathrm{A}$ humana. Enquanto executávamos os equilíbrios de oxigênio, notamos que o pico da descxiemoglobina era notavelmente mais amplo para alguns peixes que para outros, como pode ser visto na Fig. 1. Durante a análise, uma tendência tornou-se aparente. Em geral, o pico de desóxi em certos peixes é ligeiramente deslocado em direção aos comprimentos de onda mais altos em comparaçäo com $\mathrm{Hb} \mathrm{A}$. Plagioscion é representante dos perciformes; neste caso, o espectro de deóxi é quase simétrico. Os picos dos espectros de deóxi de hemoglobinas de outros peixes ficam entre os de $\mathrm{Hb} \mathrm{A}$ e Plagioscion. Não há correlação entre dependência do pico de deóxi do comprimento de onda da presença de um efeito de Rơot, mas há uma correlaçảo com parentesco filogenético: os espectros de hemoglobina dos peixes mais primitivos, como Lepidosiren e
Potamotrygon são mais semelhante à $\mathrm{Hb} \mathrm{A}$ do que aos espectros dos peixes perciformes, que são evolucionariamente mais avançados. Uma vez que o espectro de deóxi foi obtido imediatamente após a adição de ditionito, admite-se que a metaemoglobina não está contribuindo para o espectro de deóxi, uma suposição que não poderia ser feita para o espectro de óxi. No entanto, formação de hemocromógeno poderia afetar o espectro de desóxi, se ocorresse. Um argumento contra esta possibilidade é que os espectros de espécies estreitamente relacionadas são virtualmente idênticos, e isto seria improvável se hemocromógeno se formasse em quantidades variáveis. Uma investigação das diferenças espectrais relacionadas às diferenças seqüenciais ou de propriedades funcionais das hemoglobinas dos vertebrados primitivos pode provar ser muito interessante enquanto mais hemoglobinas são eventualmente sequenciadas.

Hemolisados de peixe podem conter um número variável de componentes de hemoglobina eletroforeticamente diferentes (Tabela 1; Riggs, 1970; Fyhn et al., 1978). Oma correlação entre multiplicidade de hemoglobina dos hemolisados e ocorrência ou magnitude do efeito Root foi procurada, mas não encontrada. Dentro da ordem Cypriniformes, que inclui cerca de $70 \%$ dos peixes testados, estão representadas todas as categorias de efeito Root. Os Cipriniformes desprovidos de efeito Root mostram tantas bandas de hemoglobina quantas mostram os cipriniformes com um forte efeito Root $(2,9 \pm 0,33, N=17$ e $3,4 \pm 0,43$, $\mathrm{N}=16$, respectivamente). Nenhuma correlação foi encontrada entre hemolisados de hemoglobina simples ou hemolisados de hemoglobina múltipla e a presença de um forte efeito Root ou a ausência deste efeito. Aparentemente, a multiplicidade de hemoglobina dos hemolisados não é um fator crucial para a presença de um efeito Root. Alguns hemolisados de hemoglobina simples apresentam tanto um efeito Bohr como um efeito Root (ex., Osteoglossum, Sternopygus), enquanto outros têm o eteito Bohr, mas não o Root (ex., Lepidosiren, Electrophorus). (Para presença de efeito Bohr nestes peixes, ver artigos de Galdames-Portus et al.. (1978), Garlick et al. (1978), Phelps et al. 
(1978) e Johansen et al. (1968)). A base estrutural da diferença entre o efeito Root e Bohr fica para ser demonstrada.

Em Hoplosternum littorale, em que os dois componentes foram separados (Fig. 1, E e F), um tem efeito Root e o outro um efeito Bohr reverso (ver também Garlick et al., 1978). Um padrão semelhante ocorreu em vários ou- tros ostariofísios (Mylossoma, Martin et al., 1978b; Hemiodus, Rhytiodus e Pimelodus, Bunn \& Riggs, 1978; Pterygoplichthyes, Brunori et al., 1978) . Restringir o efeito Root a certos componentes da hemoglobina pode ser uma ocorrência comum em peixes com hemoglobinas múltiplas, e parece ser uma estratégia útil. Tal compartimentação de função tem sido

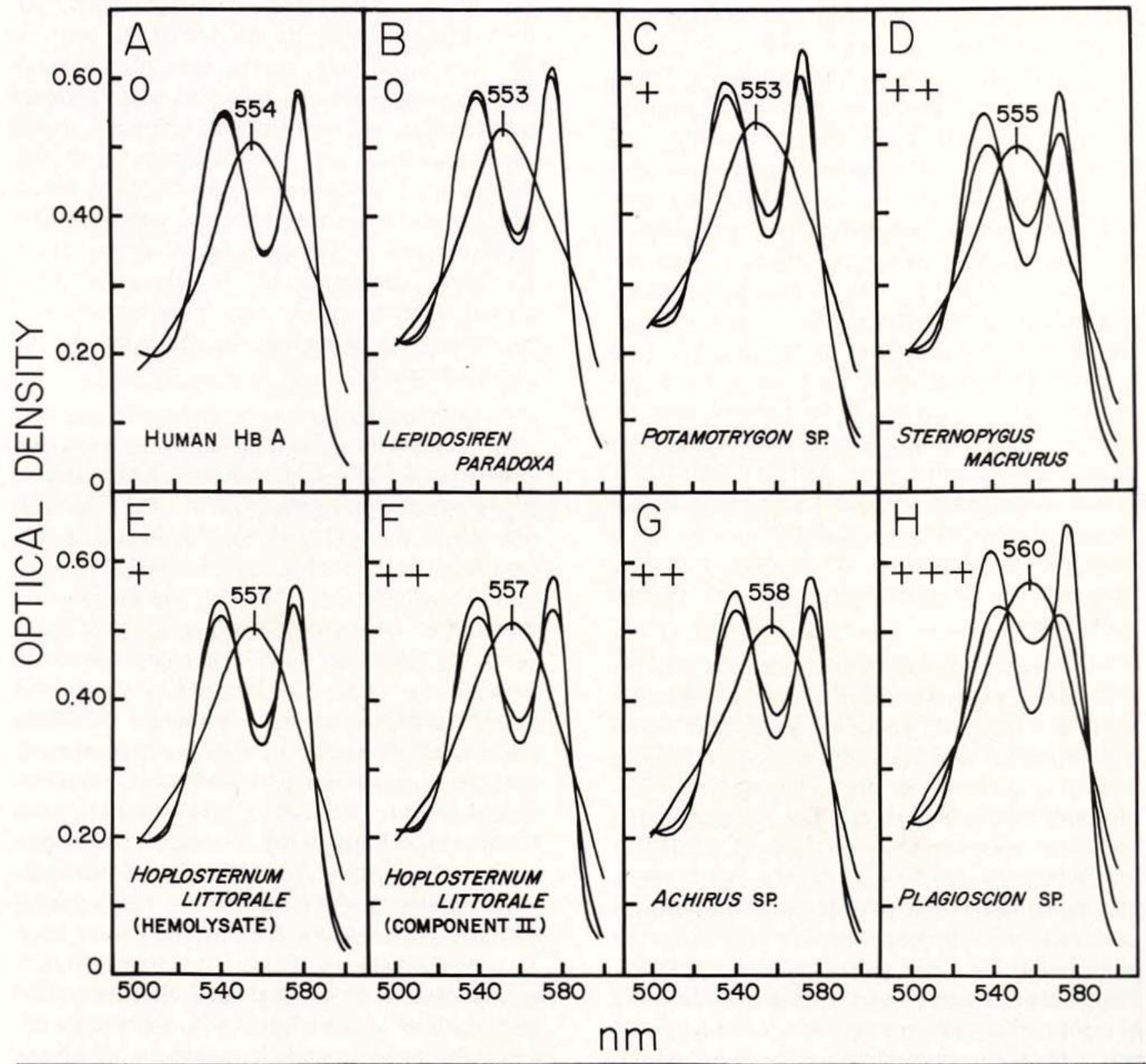

Fig. 1 - Espectros da $\mathrm{Hb}$ A humana (A) e hemolisados de cinco espécies de peixes Lepidosiren paradoxa (B); Potomotrygon sp. 4, fenótipo I (C); Sternopygus macrurus (D); Hoplosternum littorale (E); Hoplosternum littorale componente II da hemoglobina (F); Achirus sp (G); Plagioscion sp (H). Todos os espectros foram registrados a $20^{\circ} \mathrm{C}$ na presença de tampão de citrato $0,05 \mathrm{M}$. Os espectros das hemoglobinas parcialmen te desoxigenadas foram registrados a pH 5,5, espectros das óxi a pH 8,0 a 8,5 após a adição de Tris sólido, e espectros de deóxi após adição de ditionito. Uma designação de efeito Root de $\mathrm{O}$ é determinada se o hemolisa do é desoxigenado menos que $10 \%$ a pH $5,5,+$ se $10-20 \%,++$ se $20-40 \%$, e +++ se desoxigenada mais que $40 \%$. 
encontrada em vários peixes para o efeito Bohr e o efeito de temperatura (revistos por Johansen \& Weber, 1976). Presumivelmente a presença de dois diferentes tipos de hemoglobina permitiria transporte adequado de oxigênio por uma das hemoglobinas mesmo sob condições ácidas, enquanto reteria a vantagem de uma hemoglobina com efeito Root na rete mirabile do olho ou da bexiga natatória.

\section{SUMário}

Este levantamento de efeitos Root em peixes amazônicos traz à luz muitos fatos importantes. A coexistência de hemoglobinas com efeito Root e um hábito de respiração aérea, com conseqüentes tensões de $\mathrm{CO}_{2}$ sanguíneas altas em um certo número de peixes, é contrária a informações prévias, e nos força a questionar as suposições usadas para explicar estas informações. Nenhum efeito Root foi encontrado em Lepidosiren, Electrophorus ou Synbranchus, corroborando relatos prévios, mas efeitos Root pronunciados foram encontrados em Arapaima e Hoplerythrinus e efeitos Root menores em certas gimnotóides e siluróides de respiração aérea. Aparentemente, a redução na capacidade de oxigênio do sangue induzida pelo $\mathrm{CO}_{2}$ não torna 0 mecanismo de respiração aérea impraticável. São propostas duas possíveis explicações que requerem investigação posterior. Primeiro, a capacidade global de $\mathrm{O}_{2}$ pode ser maior em peixes com respiração aérea que em peixes com respiração aquática, e é reduzida pelo $\mathrm{CO}_{2}$ a um nível que é ainda adequado. Segundo, a acidose respiratória pode ser contrabalançada por uma alcalose metabólica forte, e o pH pode não ser seriamente reduzido.

Relatamos a presença de um efeito Root no hemolisado de Potamotrygon, uma arraia de água doce. Que nós saibamos, este é o primeiro relato de uma hemoglobina com efeito Root em um elasmobrânquio. No entanto, efeitos Bohr pronunciados em elasmobrânquios já haviam sido registrados anteriormente, e reconhecemos o problema semântico de separar os dois efeitos. O grau de desoxigenação do hemôlisado suficiente para ser chamado um efeito Root, e que limite mais baixo $\mathrm{pH}$, está arbitrariamente definido neste estudo como
$10 \%$ a pH 5,5. Fisiologicamente, um efeito Root mínimo pode ser inperceptivel de um efeito Bohr grande. Físico-quimicamente, a distinção entre os dois efeitos é igualmente obscura.

O padrão de ocorrência do efeito Root segue imperfeitamente linhas filogenéticas, mas há numerosas exceções que sugerem um elemento comum. A ocorrência de efeitos Root pronunciados parece correlacionar bem com a distribuição das retia mirabile da coróide dos olhos e com a distribuição da bexiga natatória bem desenvolvida. As retia mirabile da bexiga natatória, no entanto, estấo geralmente ausentes nos grupos primitivos (ostariofísios, clupeídeos) embora o efeito Root esteja presente. Possivelmente, a rete mirabile da coróide seja a mais primitiva estrutura associada à origem das hemoglobinas com efeito Root, resultando em um tipo de pré-adaptaçảo para a evolução de um eficiente mecanismo de secreção gasosa na bexiga natatória.

Dados imunológicos indicam que duas hemoglobinas podem ser significativamente diferentes em termos das propriedades de superfície, embora ambas tenham efeito Root ou vice-versa. As hemoglobinas de siluróides com efeito Root, bem como as hemoglobinas de outros ostariofísios, precipitaram com antisoro para componente I do sistema de hemoglobina da truta, uma hemoglobina sem efeito Root, mas as hemoglobinas de siluróides sem efeito Root não o fizeram.

Os espectros de deóxi de hemolisados de diferentes peixes variam significativamente. Como na reatividade imunológica cruzada, as diferenças espectrais parecem estar relacionadas com a distância evolucionária e não se correlacionam com a distribuiçăo do efelto Root. As hemoglobinas de peixe diferem espectralmente da $\mathrm{Hb}$ A humana, e os peixes mais "primativos" parecem ser menos divergentes que os peixes evolucionariamente mais "avançados".

A presença de efeitos Root nåo se correlaciona com a presença de hemoglobinas sim. ples ou múltiplas, como foi visto pelos padrões eletroforéticos para os hemolisados destes peixes. No entanto, efeitos Root parecem estar restritos a apenas certos componentes em 
vários peixes onde há presença de hemoglobinas múltiplas. Tal cơmpartimentação de função pode permitir transporte adequado de $\mathrm{O}_{2}$ através de uma grande variedade de condições ambientais.

\section{AgRAdeCIMENTOS}

A parte experimental do trabalho foi executada a bordo do R/V "Alpha Helix" e foi apoiada pela subvenção PCM75-06451 da National Science Foundation. Drs. Hans Jorgen Fyhn e Unni E.H. Fyhn agradecem o apoio da viagem do "Norwegian Research Council for Science and the Humanities". Somos gratos ao Dr. William L. Fink ("Museum of Comparative Zoology, Harvard University") pela identificação dos peixes. Martha Farmer agradece a bolsa de pesquisa da "Duke University Graduate School", No 303-3765. Apoio adicional para M. F. foi proporcionado por uma subvenção de pesquisa da NIH, HL-15460 para J. Bonaventura.

\section{SUMMARY}

The Root effect was measured in hemolysates from representatives of 56 genera of Amazonian fishes. Hemolysates from several species of airbreathing fishes were found to have Root effects, contrary to published hypotheses. Hemolysates from Potamotrygon, a fresh water ray, exhibit a Root effect under our experimental conditions. The pattern of Root effect distribution correlates positively with the distribution of choroid retia mirabile and swimbladders, but not with the distribution of swimbladder retia mirabile; it is proposed that the former is the more primitive structure which is associated with the origin of Root effect hemoglobins. Some of the fish hemoglobins differ spectrally from one another. The positions of the absorption maxima of the deoxyhemoglobins range from $553 \mathrm{~nm}$ (Lepidosiren paradoxa and Potamotrygon sp.) to $560 \mathrm{~nm}$ (Plagioscion). Occurrence of Root effects is not correlated with the complexity of the hemoglobin electrophoretic pattern, although several species are found to have multiple hemoglobin systems in which the Root effect is restricted to certain components.

\section{BIBLIOGRAFIA}

Alexander, R. MCN.

$1965 \rightarrow$ Struction in the catfish. J. Zool., 148 : 88-152.

1966 - Physical aspects of swimbladder. function. Bio. Rev., 41 : 141-176.
1967 - Functional Design in Fishes. London, Hutchinson \& Co. 160 p.

ANTONini, E.; AMIConi, G. \& BRUNori, M.

1972 - The effect of anions and cations on the oxygen equilibrium of human hemoglobin. In: Oxygen Affinity of Hemoglobins and the Red Dell Acid-base Status (Ed. M. Rorth \& P. Astrup.) Copenhagen: Munksgaard. pp. 121-129.

BeNesCh, R.; MACDuff. G. \& Benesch, R.E.

1965 - Determination of oxygen equilibria with a versatile new tonometer. Analytical Biochemistry, $11: 81-87$.

Bridge, T.W. \& Haddun, A.C.

1893 - Contribution to the anatomy of fishes - II. The airbladder and Weberian ossicles in the siluroid fishes. Phil. Trans. (B), $184: 65-333,9$ plates.

BRUNoRI, M.; BONAVENTURA, J.; FOCESI JR.; A.; GALDAMES-PORTUS, M.I. \& WILSON, M.T.

1978 - Separação e caracterização dos componentes de hemoglobina de Pterygoplichthys pardalis, o acaribodó. Acta Amazonica $8(4)$ : Suplemento. (Este número).

BUNN, H.F. \& RIGGS, A.

1978 - A medida do efeito Bohr em hemoglobinas de peixe por focalização elétrica de Gel. Acta Amazonica 8(4) : Suplemento. (Este número).

CARTER, G.S.

1931 - Aquatic and aerial respiration in animals. Biol, Rev. $6: 1-35$.

Carter, G.S. \& Beadle, L.C.

1931 - The fauna of the swamps of the Paraguayan Chaco in relation to its environment. II. Respiratory adaptations in the fishes. J. Linn. Soc. (Zool.), 37 : 327-368.

FAHLÉN, G.

1967a - Morphological aspects on the hydrostatic function of the gas bladder of Clupea harengus. Acta Univ. Lund. Sect., 2(1) : 49 .

$1967 \mathrm{~b}$ - Morphology of the gas bladder of Coregonus larvaretus L. Acta. Univ. Lund. Sect., 2(28):37.

FANGE, $R$.

1958 - The structure and function of the gas bladder in Argentina silus. Quart. J. Microscop. Sci., 99 : 95-102.

1966 - The physiology of the swimbladder. Physiol. Rev., 46 : 299-322.

FINK, W.L. \& FINK, S.V.

1978 - A Amazônia Central e seus peixes. Acta Amazonica 8(4): Suplemento. (Este número). 
FISH, G.R.

1956 - Some aspects of the respiration of $\mathbf{s i x}$ species of fish from Uganda. J. Exp. Biol., 33 : 185195.

FYHN, U.E.H.; FYHN, H.J.; DAvIS, B.J.; POWERs, D.A.; FINK, W.L. \& GARLICK, R.L.

1978 - Heterogeneidade de hemoglobinas nos peixes da Amazônia. Acta Amazonica 8(4) : Suplemento. (Este número).

Galdames-Portus, M.I.; Noble, R.W.; Farmer, M.; POWERS, D.A ; BRUNORI, M.; FYHN, H.J. \& FYHN, U.E.H.

1978 - Estudos das propriedades funcionais das hemoglobinas de Osteoglossum bi cirrhosum e Arapaima gigas. Acta Amazonica 8(4):Suplemento. (Este número).

GARLICK, R.L.; BUNN, H.F.; FYHN, H.J.; FYHN, U.E.H; Martin, J.P.; Noble, R.W. \& Powers, D.A.

1978 - Estudos funcionais na hemoglobina de componentes separados de um bagre de respiração aérea, Hoplosternum littora le (Hancock). Acta Amazonica 8(4): Suplemento. (Este número).

GREENWOOD, P.H.; ROSEN, D.E.; WeItZMAN, S.H. \& MYeRs, G.S.

1966 - Phyletic studies of teleostean fishes, with a provisional classification of living forms. Bull. Am. Mus. Nat. Hist., $131: 339-455$.

JOHANSEN, $\mathbf{K}$.

1966 - Airbreathing in the teleost Symbranchus marmoratus. Comp. Biochem. Physiol., $18: 383-395$.

1968 - Air-breathing fishes. Sci. Amer., 219 : 102-111.

1970 - Air breathing in fishes. In: Fish Physio. logy (ed. Hoar, W.S. \& Randall, D.J.). New York, Academic Press. v. 4:361-411.

JoHANSEN, K. \& LENFANT, C.

1967 a - Respiratory function in the South American Lungfish, Lepidosiren paradoxa (Fitz) J. Exp. Biol., $46: 205-218$.

1967b-Respiratory adaptations in selected amphibians. Resp. Physiol., 2 : 247-260.

Johansen, K.; LeNfant, C.; SChMidT-Nielsen, $\mathbf{K}$. \& Petersen, J.A.

1968 - Gas exchange and control of breathing in the electric eel, Electrophorus electricus. Z. Vergl. Physiol., 61:137-163.

JOHANSEN, K. \& WEBER, R.E.

1976 - On the adaptability of haemoglobin function to enviromental conditions. In: Perspectives in Experimental Biology (ed. David, P.S.) Zoology. Oxford, Pergamon Press. 1 : 219-234.

JoNes, F.R.H. \& MARShalL, N.B.

1953 - The structure and functions of the teleostean swimbladder. Biol. Rev., 28 : $16-83$
KRAMER, D.L. \& GrAinM, J.B.

1976 - Synchroncus air breathing, a social ccmponent of respiration in fishes. Copeia, pp. 689-697.

KROGH, A. \& LeITCH, 1.

1919 - The respiratory function of the blood in fishes. J. Physiol., $52: 288-300$.

Kuhn, W.; Kamel, A., KuhN, H.J. \& Marti, E.

1963 - The filling mechanism of the swimbladder. Generation of high gas pressures through hairpin countercurrent :nultiplication. Experentia, 19:497-552.

LENFANT, C. \& JohANSEN. K.

1968 - Respiration in the African lungfish Protopterus aethiopicus. I. Respiratory properties of blood and normal patterns of breathing and gas exchange. J. Exp. Biol., 49 : 437-452.

Lenfant, C.; Johansen, K. \& Grigg, G.C.

1966 - Respiratory properties of blood and pattern of blood and pattern of gas exchange in the exchange in the lungfish, Neoceratodus forsteri (Krefft) Resp. Physiol., 2 : 1-21.

LOWE-MCCONNELL, R.H.

1975 - Fish communitics in tropical Freshwaters. New York, Longman, $337 \mathrm{p}$.

MANWELL, C.

1958 - Ontogeny of hemoglobin in the Skate Raja, binoculata. Science, $128: 419-420$.

1963 - Fetal and Adult hemoglobin of the Spiny Dogfish Squalus suckleyi. Arch. Biochem. Biophys, 101 : 504-511.

MARTIN, J.P.; BONAVENTURA, J.; BRUNORI, M.; FyhN, H.J.; FYHN, U.E.H.; GARLICK, R.L.; POWERS, D.A. \& WILSON, M.T.

1973 - The isolation and characterization of the hemoglobin components of Mylossoma sp., an Amazonian teleost. Comp. Biochem. Physiol. (this issue)

MARTIN, J.P.; BONAVENTURA, J.; FYhN, H.J.; FyHN, U.E.H.; Garlick, R.L. \& Powers, D.A.

1978 - Estudo da estrutura e função das hemoglobinas isoladas das arraias da Amazônia do gênero Potamotrygon. Acta Amazonica 8(4): Suplemento. (Este número).

NeLsON, J.S

1976 - Fishes of the world. New York, John Wiley. $416 \mathrm{p}$.

Phelps, C.; FARMer, M.; Fyhn, H.J.; Fyhn, U.E.H.; GARLICK, R.L.; Noble, R.W. \& Powers, D.A.

1978 - Equilíbrio e cinética de união de oxigê. nio e monóxido de carbono à hemoglobina do peixe pulmonado sul americano Lepidosiren paradoxa. Acta Amazonica 8(4) : Suplemento. (Este número). 
POGGENDORF, D.

1952 - Die absoluten Horschwellen des Zwerg welses (Ameriurus nebulosus) und Beitrage zur Physik des Weberschen Apparates der Ostariophysen. Z. Vergl. Physiol., 34 : 222-257.

RAHN, H.

- GAS transport from the external environment to the cell. In: Ciba Foundation Symposium on Development of the Lung (Ed. A.V.S. de Reuck \& R. Porter) London, J. \& A. Churcill Ltd. pp. 3-23.

REICHLIN, M. \& DAvis, B.J.

1978 - Relações antigênicas entre peixes co. muns à bacia do rio Amazonas. Acta Amazonica 8(4) : Suplemento. (Este número).

RIGGS, A.

1970 - Properties of fish hemoglobins. In: Fish Physiology. (Ed. W.S. Hoar. \& D.J. Randall) New York, Academic Press. v. $4: 209-251$.

RIggs, A.; Fyhn, H.J.; FyHN, U.E.H. \& Noble, R.W. 1978 - Estudos das propriedades funcionais da hemoglobina de Hoplias malabaricus e
Hoplerythrinus unitaeniatus. Acta Ama zonica $8(4)$ : Suplemento. (Este número).

Roor, R.W.

1931 - The respiratory function of the blood of marine fishes. Biol. Bull., 61 : 427-456.

STEEN, J.B.

1963 - The physiollogy of the swimbladder in the eel Anguilla vulgaris. III. The mechanism of gas secretion. Acta Physiol. Scand, 59 : 221-241.

WILLMER, E.N.

1934 - Some observations on the respiration of certain tropical fresh-water fishes. J. Exp. Biol. 11 : 283-306.

WitTenberg, J.B. \& Haedrich, R.L.

1974 - The choroid rete mirabile of the fish eya. II. Distribution and relation to the pseudobranch and to the swimbladder rete mirabile. Biol. Bull., 146 : $137-156$.

Wittenberg, J.B. \& Wittenberg, B.A.

1974 - The choroid rete mirabile of the fish eye. I. Oxygen secretion and structure: comparison with the swimbladder rete mirabile. Biol. Bull. 146 : 116-136. 